\title{
SOME LOG-MAJORIZATIONS AND AN EXTENSION OF A DETERMINANTAL INEQUALITY
}

\author{
RUTE LEMOS AND GRAÇA SOARES
}

\begin{abstract}
An eigenvalue inequality involving a matrix connection and its dual is established, and some log-majorization type results are obtained. In particular, some eigenvalues inequalities considered by F. Hiai and M. Lin [9], an associated conjecture, and a singular values inequality by L. Zou [20] are revisited. A reformulation of the inequality $\operatorname{det}\left(A+U^{*} B\right) \leq \operatorname{det}(A+B)$, for positive semidefinite matrices $A, B$, with $U$ a unitary matrix that appears in the polar decomposition of $B A$, is also extended, using some known norm inequalities, associated to Furuta inequality and Araki-Cordes inequality.
\end{abstract}

\section{INTRODUCTION}

Let $\mathbf{x}=\left(x_{1}, \ldots, x_{n}\right), \mathbf{y}=\left(y_{1}, \ldots, y_{n}\right) \in \mathbb{R}^{n}$ be vectors with the components sorted in nonincreasing order, that is, $x_{1} \geq \cdots \geq x_{n}$ and $y_{1} \geq \cdots \geq y_{n}$. We say that $\mathbf{y}$ weakly majorizes $\mathbf{x}$ and write $\mathbf{x} \prec_{w} \mathbf{y}$ if

$$
\sum_{i=1}^{k} x_{i} \leq \sum_{i=1}^{k} y_{i}, \quad k=1, \ldots, n .
$$

If $\mathbf{x} \prec_{\mathrm{w}} \mathbf{y}$ and equality holds in (1) for $k=n$, we say that $\mathbf{y}$ majorizes $\mathbf{x}$, denoted by $\mathbf{x} \prec \mathbf{y}$. For $\mathbf{x}, \mathbf{y}$ with nonnegative components, we write $\mathbf{x} \prec_{\log } \mathbf{y}$ if $\mathbf{y} \log$-majorizes $\mathbf{x}$, that is,

$$
\prod_{i=1}^{k} x_{i} \leq \prod_{i=1}^{k} y_{i}, \quad k=1, \ldots, n,
$$

with equality occurring in (2) when $k=n$.

For any real valued function $f$ defined on an interval, containing all the components of the real vector $\mathbf{x}$, we adopt the notation $f(\mathbf{x})=\left(f\left(x_{1}\right), \ldots, f\left(x_{n}\right)\right)$. If all the components of $\mathbf{x}, \mathbf{y}$ are positive, then $\mathbf{x} \prec_{\log } \mathbf{y}$ if and only if $\log \mathbf{x} \prec \log \mathbf{y}$, this justifying the $\log$-majorization terminology. If $f$ is convex, then $\mathbf{x} \prec \mathbf{y}$ implies $f(\mathbf{x}) \prec_{\mathrm{w}} f(\mathbf{y})$. In particular, the log-majorization implies the weak majorization. Additionally, if $f$ is an increasing and convex function, then $\mathbf{x} \prec_{\mathrm{w}} \mathbf{y}$ implies $f(\mathbf{x}) \prec_{\mathrm{w}} f(\mathbf{y})$. For instance, $f(t)=\ln \left(1+e^{t}\right)$ is a strictly increasing and convex function on $(0,+\infty)$. Two important resources on the topic of majorization are $[2,15]$.

Let $M_{n}$ be the algebra of $n \times n$ complex matrices and $I$ be the identity matrix of order $n$. For $A \in M_{n}$ with real eigenvalues, we denote by $\lambda(A)$ the $n$-tuple of eigenvalues of $A$ arranged as follows $\lambda_{1}(A) \geq \cdots \geq \lambda_{n}(A)$. If $A, B \in M_{n}$, then $A B$ and $B A$ have the same eigenvalues, including multiplicities [11, Theorem 1.3.20], hence $\lambda(A B)=\lambda(B A)$.

For simplicity of notation, if $A, B \in M_{n}$ have real eigenvalues, then we write $A \prec_{\mathrm{w}} B$ whenever $\lambda(A) \prec_{\mathrm{w}} \lambda(B)$; moreover, if $A, B \in M_{n}$ have nonnegative eigenvalues, we write $A \prec_{\log } B$ when $\lambda(A) \prec_{\log } \lambda(B)$. Majorization is a powerful tool for establishing determinantal and matrix norm inequalities. In particular, if $A \prec_{\log } B$, then $\operatorname{det}(I+A) \leq \operatorname{det}(I+B)$. On the other hand, some classical determinantal inequalities can find their majorization counterparts.

Key words and phrases. Log-majorization; matrix connections and means; eigenvalues; singular values; trace and determinantal inequalities; Löwner-Heinz inequality; Furuta inequality; Araki-Cordes inequality

2010 Mathematics Subject Classification. 15A42; 47A63; 47A64; 47A30 . 
For $A \in M_{n}$, the unique positive semidefinite square root of $A^{*} A$ is denoted by $|A|$. For $A, B \in M_{n}$, Ky Fan Dominance Theorem [15] asserts that $|A| \prec_{\mathrm{w}}|B|$ if and only if $\|A\| \leq\|B\|$ holds for any unitarily invariant norm $\|\cdot\|$ in $M_{n}$. We recall that a norm $\|\cdot\|$ is said to be unitarily invariant in $M_{n}$ if $\|U A V\|=\|A\|$ for all $A \in M_{n}$ and all unitary matrices $U, V \in M_{n}$. Considering the singular values of $A \in M_{n}$, that is, the eigenvalues of $|A|$, ordered as follows $s_{1}(A) \geq \cdots \geq s_{n}(A)$, the Ky Fan $k$-norms of $A$ defined by

$$
\|A\|_{(k)}=\sum_{i=1}^{k} s_{i}(A), \quad 1 \leq k \leq n,
$$

including the spectral (or operator) norm $\|A\|$, when $k=1$, are examples of unitarily invariant norms in $M_{n}$.

As usual, $A \geq B$ means that $A, B \in M_{n}$ are Hermitian and $A-B$ is positive semidefinite; $A>0$ means that $A \in M_{n}$ is Hermitian and positive definite. Let $0 \leq \alpha \leq 1$. The famous Löwner-Heinz inequality [14] states that $A \geq B \geq 0$ implies $A^{\alpha} \geq B^{\alpha}$. Kubo and Ando [12] introduced the $\alpha$-power mean of positive semidefinite matrices $A, B$ as

$$
A \sharp_{\alpha} B=A^{\frac{1}{2}}\left(A^{-\frac{1}{2}} B A^{-\frac{1}{2}}\right)^{\alpha} A^{\frac{1}{2}},
$$

when $A$ is invertible, and extended to any non-invertible $A$ by continuity as follows:

$$
A \sharp_{\alpha} B=\lim _{\epsilon \rightarrow 0^{+}}(A+\epsilon I) \sharp_{\alpha} B .
$$

If $A B=B A$, then $A \sharp_{\alpha} B=A^{1-\alpha} B^{\alpha}$. In general, $A \sharp_{1-\alpha} B=B \sharp_{\alpha} A$ and $(A, B) \mapsto A \sharp_{\alpha} B$ is jointly monotone, as a consequence of Löwner-Heinz inequality. In particular, $\sharp=\sharp_{1 / 2}$ denotes the geometric mean. We recall that $A \sharp B$ is the unique positive solution of the Riccati equation $X A^{-1} X=B$, also characterized by Pusz and Woronowicz [18] as

$$
A \sharp B=\max \left\{X \geq 0:\left[\begin{array}{cc}
A & X \\
X & B
\end{array}\right] \geq 0\right\} .
$$

This note is organized as follows. In Section 2, recalling the Kubo-Ando axiomatic theory of matrix connections [12], an eigenvalue inequality is established, involving a matrix connection and its dual. In Section 3, the antisymmetric tensor power technique is used to prove some log-majorizations. As a consequence, previous known results are revisited. In particular, an eigenvalues inequality, involving the $\alpha$-power mean, considered by F. Hiai and M. Lin [9, Theorem 2.5], as well as a singular value inequality for the geometic mean due to L. Zou [20, Theorem 2.10] are reobtained. A conjecture, considering eigenvalues replaced by singular values, in the same spirit of the one presented in [9, Conjecture 2.6] is also raised. In Section 4, a reformulation of the determinantal inequality

$$
\operatorname{det}\left(A+U^{*} B\right) \leq \operatorname{det}(A+B),
$$

for positive semidefinite matrices $A, B$, where $U$ is a unitary matrix that appears at the polar decomposition of $B A$, formulated by K. M. R. Audenaert [1], when comparing geodesics induced by different metrics, and further complemented by M. Lin [13], is extended. Such an extension is obtained, using the interplay between majorization relations and determinantal inequalities. The main tools are a norm inequality [17, Theorem 1], which is a simultaneous extension of Araki-Cordes inequality [5] and Bebiano-Lemos-Providência inequality [4], and its reverse [17, Theorem 2], as well as another inequality for unitarily invariant norms obtained via logmajorization [7] from Furuta inequality [6]. 


\section{Eigenvalue inequality FOR MATRIX CONNECTIONS}

The axiomatic theory of connections and means for pairs of positive operators was developed by F. Kubo and T. Ando [12]. A binary operation $\sigma$ on the cone of $n \times n$ positive semidefinite matrices, satisfying for all $A, B, C, D \geq 0$ the following conditions:

C1. (joint monotonicity) $A \leq C$ and $B \leq D$ imply $A \sigma B \leq C \sigma D$;

C2. (transformer inequality) $X^{*}(A \sigma B) X \leq\left(X^{*} A X\right) \sigma\left(X^{*} B X\right)$ for $X \in M_{n}$;

C3. (joint continuity from above) for $A_{n}, B_{n} \geq 0$, if $A_{n} \downarrow A$ and $B_{n} \downarrow B$, then $A_{n} \sigma B_{n} \downarrow A \sigma B$ is called a (matrix) connection. A (matrix) mean is a connection $\sigma$, satisfying $I \sigma I=I$.

For each connection $\sigma$, there exists a unique operator monotone function $f: \mathbb{R}^{+} \rightarrow \mathbb{R}^{+}$, such that $f(t) I=I \sigma(t I), t \in \mathbb{R}^{+}$. Such function $f$ is called the representing function of $\sigma$. The formula

$$
A \sigma B=A^{\frac{1}{2}} f\left(A^{-\frac{1}{2}} B A^{-\frac{1}{2}}\right) A^{\frac{1}{2}}
$$

holds for $A>0, B \geq 0$, where the right hand side is defined via the analytic functional calculus, and it can be extended to $A \geq 0$ by continuity as follows:

$$
A \sigma B=\lim _{\epsilon \rightarrow 0^{+}}(A+\epsilon I) \sigma B .
$$

The dual of a connection $\sigma$ is the connection $\sigma^{\perp}$ defined for $A, B>0$ by

$$
A \sigma^{\perp} B=\left(B^{-1} \sigma A^{-1}\right)^{-1}
$$

and extended by continuity to $A, B \geq 0$ as usual. Since $t f(t)^{-1}$ is the representing function of $\sigma^{\perp}$, when the representing function of $\sigma$ is $f(t)$, it is clear that

$$
(A \sigma B) X\left(A \sigma^{\perp} B\right) X \quad \text { and } \quad A X B X
$$

have the same determinant for any $X \in M_{n}$. If $\sigma$ is the right trivial mean, then its dual is the left trivial mean and the matrices (3) are trivially equal. An easy consequence of the properties (C1)-(C3) of the definition of $\sigma$ is the next inequality between the maximum eigenvalue of the matrices (3) for any $A, B, X \geq 0$.

Theorem 2.1. Let $A, B, X \geq 0$ and $\sigma$ be a connection. Then

$$
\lambda_{1}\left((A \sigma B) X\left(A \sigma^{\perp} B\right) X\right) \leq \lambda_{1}(A X B X) .
$$

Proof. Firstly, let $A, B>0$. If $X=I$, then we only need to show that $\lambda_{1}(A B) \leq 1$ implies

$$
\lambda_{1}\left((A \sigma B)\left(A \sigma^{\perp} B\right)\right) \leq 1,
$$

because both sides of (4) have the same order of homogeneity for $A, B$, so that we can multiply $A, B$ by a positive scalar. Since $A, B$ are invertible, from $\lambda_{1}(A B) \leq 1$, we have $A \leq B^{-1}$, as well as $B \leq A^{-1}$. By the joint monotonicity of $\sigma$, we get

$$
A \sigma B \leq B^{-1} \sigma A^{-1}=\left(A \sigma^{\perp} B\right)^{-1},
$$

consequently, (5) holds. Now, let $X>0$. The transformer inequality (C2) becomes an equality, when $X$ is invertible. Thus, denoting $X^{\frac{1}{2}} A X^{\frac{1}{2}}$ by $A_{X}$, we have

$$
\lambda_{1}\left((A \sigma B) X\left(A \sigma^{\perp} B\right) X\right)=\lambda_{1}\left(\left(A_{X} \sigma B_{X}\right)\left(A_{X} \sigma^{\perp} B_{X}\right)\right) \leq \lambda_{1}\left(A_{X} B_{X}\right)=\lambda_{1}(A X B X) .
$$

When $A, B, X$ are not invertible, we may replace $A, B, X$ in (4) by $A+\epsilon I, B+\epsilon I, X+\epsilon I>0$, for $\epsilon>0$, respectively, and then we use a continuity argument, letting $\epsilon \downarrow 0$. 
Corollary 2.2. Let $A, B \geq 0$ such that $A+B$ is invertible. Then

$$
\lambda_{1}\left((A+B) A(A+B)^{-1} B\right) \leq \lambda_{1}(A B),
$$

occurring equality when $A B=B A$.

Proof. If $A, B, A+B$ are invertible and $A \sigma B=A+B$, then

$$
A \sigma^{\perp} B=\left(A^{-1}+B^{-1}\right)^{-1}=A(A+B)^{-1} B
$$

and the required eigenvalue inequality readily follows from Theorem 2.1 for $X=I$. If $A, B \geq 0$ and either $A$ or $B$ is not invertible, then $A_{\epsilon}=A+\epsilon I, B_{\epsilon}=B+\epsilon I, \epsilon>0$, are positive definite and the result is obtained by a continuity argument. If $A B=B A$, then $(A+B) A=A(A+B)$ or, equivalently,

$$
(A+B) A(A+B)^{-1} B=A B,
$$

that is, the eigenvalue inequality occurs as equality.

In general, the following eigenvalues inequalities do not hold

$$
\lambda_{i}\left((A+B) A(A+B)^{-1} B\right) \leq \lambda_{i}(A B), \quad i=2, \ldots, n,
$$

as the following counterexample shows.

Example Consider the positive definite matrices

$$
A=\left[\begin{array}{ccc}
2 & -1 & 0 \\
-1 & 2 & -1 \\
0 & -1 & 2
\end{array}\right], \quad B=\left[\begin{array}{lll}
4 & 0 & 0 \\
0 & 1 & 0 \\
0 & 0 & 1
\end{array}\right]
$$

The eigenvalues of $(A+B) A(A+B)^{-1} B$ are

$$
7.9161, \quad 2.7485, \quad 0.7354
$$

while the eigenvalues of $A B$ are

$$
8.6185, \quad 2.6918, \quad 0.6897 .
$$

\section{SOME LOG-MAJORIZATION RESUlTS}

For $k=1, \ldots, n$ and $n_{k}=\left(\begin{array}{l}n \\ k\end{array}\right)$, we denote the $k$ th compound or $k$ th antisymmetric tensor power of $A \in M_{n}$ by $A^{\wedge k}$, that is, the matrix in $M_{n_{k}}$ with entries given by the minors $\operatorname{det} A(\mathbf{i}, \mathbf{j})$, where the index sets $\mathbf{i}, \mathbf{j} \subset\{1, \ldots, n\}$ have cardinality $k$ and are lexicographically ordered. As usual, $A(\mathbf{i}, \mathbf{j})$ denotes the submatrix of $A$ that lies in rows and columns indexed, respectively, by $\mathbf{i}, \mathbf{j}$. We list some essential properties of these matrices [2] for $A, B \in M_{n}$ :

P1. $(A B)^{\wedge k}=A^{\wedge k} B^{\wedge k}$ (Binet-Cauchy formula);

P2. $\left(A^{\wedge k}\right)^{r}=\left(A^{r}\right)^{\wedge k}, r>0$, and if $A$ is invertible, then $\left(A^{\wedge k}\right)^{-1}=\left(A^{-1}\right)^{\wedge k}$.

Hence, any expression involving products and fractional matrix powers "commutes" with the $k$ th antisymmetric tensor power. Moreover, $\lambda_{i}\left(A^{\wedge k}\right)=\prod_{j=1}^{k} \lambda_{i_{j}}(A), 1 \leq i_{1}<\cdots<i_{k} \leq n$ holds, so that

P3. $\left\|A^{\wedge k}\right\|=s_{1}\left(A^{\wedge k}\right)=\prod_{i=1}^{k} s_{i}(A), k=1, \ldots, n$.

If $A, B \in M_{n}$ have nonnegative eigenvalues, it follows that

P4. $A \prec_{\log } B$ if and only if $\operatorname{det} A=\operatorname{det} B$ and $\lambda_{1}\left(A^{\wedge k}\right) \leq \lambda_{1}\left(B^{\wedge k}\right), k=1, \ldots, n$. 
In this section, we illustrate the potential of using the antisymmetric tensor power technique to derive some log-majorizations and as a consequence some known results are revisited.

The next result is a corollary of Theorem 2.1.

Corollary 3.1. If $A, B, X \geq 0$ and $\alpha \in[0,1]$, then

$$
\left(A \sharp_{\alpha} B\right) X\left(A \sharp_{1-\alpha} B\right) X \prec_{\log } A X B X \text {. }
$$

Proof. Theorem 2.1 holds with $A, B, X$ replaced by their $k$ th compounds $A^{\wedge k}, B^{\wedge k}, X^{\wedge k} \geq 0$ for any connection $\sigma$ and each $k=1, \ldots, n$. In particular, if $\sigma=\sharp_{\alpha}$, then $\sigma^{\perp}=\sharp_{1-\alpha}$. By properties $\mathrm{P} 1$ and $\mathrm{P} 2$, we have

$$
\begin{gathered}
\left(A^{\wedge k} \sharp_{\alpha} B^{\wedge k}\right) X^{\wedge k}\left(A^{\wedge k} \sharp_{1-\alpha} B^{\wedge k}\right) X^{\wedge k}=\left(\left(A \sharp_{\alpha} B\right) X\left(A \sharp_{1-\alpha} B\right) X\right)^{\wedge k}, \\
A^{\wedge k} X^{\wedge k} B^{\wedge k} X^{\wedge k}=(A X B X)^{\wedge k},
\end{gathered}
$$

$k=1, \ldots, n$. Then the required log-majorization holds, recalling the equality between the determinants of the matrices in (6) and using $\mathrm{P} 4$.

The log-majorization (6) may be equivalently formulated, for $A, B, X \geq 0$ and $\alpha \in[0,1]$, by

$$
\prod_{i=k}^{n} \lambda_{i}\left(\left(A \sharp_{\alpha} B\right) X\left(A \sharp_{1-\alpha} B\right) X\right) \geq \prod_{i=k}^{n} \lambda_{i}(A X B X), \quad k=1, \ldots, n,
$$

with equality for $k=n$. When $X=I$, these inequalities are included in [9, Theorem 2.5] considered by F. Hiai and M. Lin.

For $s, t \in \mathbb{R}$ and $A, B>0$, for simplicity of notation, we will consider

$$
A \natural_{s, t} B=A^{\frac{s}{2}}\left(A^{-\frac{1}{2}} B A^{-\frac{1}{2}}\right)^{t} A^{\frac{s}{2}},
$$

extended to $A, B \geq 0$ by continuity as usual. For $0 \leq \alpha \leq 1$, we have $A \natural_{1, \alpha} B=A \sharp_{\alpha} B$.

If $A, B \geq 0$, then Corollary 3.1 with $X=A^{\frac{s+r}{2}-1}, r, s \in \mathbb{R}$ and $t \in[0,1]$ yields

$$
\left(A \natural_{r, t} B\right)\left(A \natural_{s, 1-t} B\right) \prec_{\log } A^{r+s-1} B .
$$

It is natural to ask if eigenvalues may be replaced by singular values in the inequalities of the previous log-majorization as Hiai and Lin [9, Conjecture 2.6] did for (7) when $X=I$, proving it in case $t \in\left[\frac{1}{4}, \frac{3}{4}\right]$. In a similar way, the following conjecture can be formulated.

Conjecture. If $A, B \geq 0, r, s \in \mathbb{R}$ and $0 \leq t \leq 1$, then

$$
\left|\left(A \natural_{r, t} B\right)\left(A \natural_{s, 1-t} B\right)\right| \prec_{\log }\left|A^{r+s-1} B\right| .
$$

We will prove the conjecture in a particular case, using the next proposition.

Proposition 3.2. Let $A, B>0, r, s \geq 0$ and $\frac{r}{r+s} \leq 2 t \leq \frac{2 r+s}{r+s}$. If $B \leq A^{1-r-s}$, then

$$
\left(A \natural_{s, 1-t} B\right)^{2} \leq\left(A \natural_{r, t} B\right)^{-2} .
$$

Proof. Let $C=A^{-\frac{1}{2}} B A^{-\frac{1}{2}}$. Then

$$
\left(A \natural_{r, t} B\right)\left(A \natural_{s, 1-t} B\right)=A^{\frac{r}{2}} C^{t} A^{\frac{r+s}{2}} C^{1-t} A^{\frac{s}{2}} .
$$


From $B \leq A^{1-r-s}$, we have $C \leq A^{-(r+s)}$ and $A^{r+s} \leq C^{-1}$. Using Löwner-Heinz inequality, under the hypothesis that $r, s \geq 0$ and $\frac{r}{r+s} \leq 2 t \leq \frac{2 r+s}{r+s}$, this two last inequalities imply

$$
\begin{array}{rlrl}
\left(A \natural_{r, t} B\right)\left(A \natural_{s, 1-t} B\right)^{2}\left(A \natural_{r, t} B\right) & =A^{\frac{r}{2}} C^{t} A^{\frac{r+s}{2}} C^{1-t} A^{s} C^{1-t} A^{\frac{r+s}{2}} C^{t} A^{\frac{r}{2}} & \\
& \leq A^{\frac{r}{2}} C^{t} A^{\frac{r+s}{2}} C^{2(1-t)-\frac{s}{r+s}} A^{\frac{r+s}{2}} C^{t} A^{\frac{r}{2}}, & 0 \leq \frac{s}{r+s} \leq 1 \\
& \leq A^{\frac{r}{2}} C^{t} A^{2(r+s)(t-1)+s} C^{t} A^{\frac{r}{2}}, & & 0 \leq 2(1-t)-\frac{s}{r+s} \leq 1 \\
& \leq A^{\frac{r}{2}} C^{\frac{r}{(r+s)}} A^{\frac{r}{2}}, & & 0 \leq 2 t-1+\frac{s}{r+s} \leq 1 \\
& \leq A^{\frac{r}{2}} A^{-r} A^{\frac{r}{2}}, & & 0 \leq \frac{r}{r+s} \leq 1 \\
& =I . & &
\end{array}
$$

Hence, (8) occurs.

Remark. It follows from the previous proposition that

$$
\left\|\left(A \natural_{r, t} B\right)\left(A \natural_{s, 1-t} B\right)\right\| \leq\left\|A^{r+s-1} B\right\|
$$

holds for $r, s \geq 0$ and $\frac{r}{r+s} \leq 2 t \leq 1-\frac{s}{r+s}$. In fact, if $\left\|A^{r+s-1} B\right\| \leq 1$, then $B^{2} \leq A^{2(1-r-s)}$. By Löwner-Heinz inequality, we have $B \leq A^{1-r-s}$, this implying

$$
\left\|\left(A \natural_{r, t} B\right)\left(A \natural_{s, 1-t} B\right)\right\| \leq 1,
$$

by Proposition 3.2. Replacing the matrices in (9) by their $k$ th compounds, by properties P1, P2 and P3, we may confirm the conjecture in that special case of $r, s, t$. In particular, if $r+s=2$, then $\frac{r}{4} \leq t \leq 1-\frac{s}{4}$. The case confirmed by Hiai and Lin occurs when $r=s=1$.

Theorem 3.3. If $A, B \geq 0$, then

$$
A(A \sharp B) B(A \sharp B) \prec_{\log } A^{2} B^{2} .
$$

Proof. Let $A, B \geq 0$. It is clear that $A(A \sharp B) B(A \sharp B)$ and $A^{2} B^{2}$ have the same determinant. Assuming $A, B$ invertible, let us prove that

$$
\lambda_{1}(A(A \sharp B) B(A \sharp B)) \leq \lambda_{1}\left(A^{2} B^{2}\right) .
$$

If $\lambda_{1}\left(A^{2} B^{2}\right) \leq 1$, then $B^{2} \leq A^{-2}$. By Löwner-Heinz inequality, we have $B \leq A^{-1}$. Therefore

$$
(A \sharp B) B(A \sharp B) \leq(A \sharp B) A^{-1}(A \sharp B)=B \leq A^{-1},
$$

because $(A \sharp B) A^{-1}(A \sharp B)=B$. We conclude that

$$
\lambda_{1}(A(A \sharp B) B(A \sharp B)) \leq 1 .
$$

If $A, B$ are not invertible, we may use a continuity argument, replacing $A$ by $A+\epsilon I$ and $B$ $B+\epsilon I$ to obtain (10). By properties P1 and P2, we have $\left(A^{\wedge k}\right)^{2}\left(B^{\wedge k}\right)^{2}=\left(A^{2} B^{2}\right)^{\wedge k}$ and

$$
(A(A \sharp B) B(A \sharp B))^{\wedge k}=A^{\wedge k}\left(A^{\wedge k} \sharp B^{\wedge k}\right) B^{\wedge k}\left(A^{\wedge k} \sharp B^{\wedge k}\right) .
$$

Finally, the result follows from inequality (10) applied to the matrices $A^{\wedge k}, B^{\wedge k}, k=1, \ldots, n$, using property $\mathrm{P} 4$.

We remark that the singular values of $A^{\frac{1}{2}}(A \sharp B) B^{\frac{1}{2}}$ and $A B$ coincide with the square roots of the eigenvalues of $A(A \sharp B) B(A \sharp B)$ and $A^{2} B^{2}$, respectively. Hence, we have the following easy consequence of Theorem 3.3.

Corollary 3.4. If $A, B \geq 0$, then $\left|A^{\frac{1}{2}}(A \sharp B) B^{\frac{1}{2}}\right| \prec_{\log }|A B|$. 
Proof. The log-majorization in Theorem 3.3 can be equivalently formulated as

$$
B^{\frac{1}{2}}(A \sharp B) A(A \sharp B) B^{\frac{1}{2}} \prec_{\log }|A B|^{2}
$$

and the matrix in the LHS of the previous log-majorization is $\left|A^{\frac{1}{2}}(A \sharp B) B^{\frac{1}{2}}\right|^{2}$. Now the result follows, because a log-majorization between two positive semidefinite matrices implies the corresponding log-majorization between the square roots of such matrices.

Corollary 3.4 contains the singular values inequalities

$$
\prod_{i=1}^{k} s_{i}\left(A^{\frac{1}{2}}(A \sharp B) B^{\frac{1}{2}}\right) \leq \prod_{i=1}^{k} s_{i}(A B), \quad k=1, \ldots, n,
$$

proved by L. Zou [20, Theorem 2.10], using a different approach.

Inspired by the previous result, it is natural to ask if the following log-majorization holds:

$$
\left|A^{\alpha}\left(A \sharp_{\alpha} B\right) B^{1-\alpha}\right| \prec_{\log }|A B|,
$$

for $A, B \geq 0$ and $\alpha \in[0,1]$, being the case $\alpha=\frac{1}{2}$ presented in Corollary 3.4.

\section{An EXTENSION OF A DETERMinANTAL INEQUALITY}

A. Matsumoto, R. Nakamoto and M. Fujii [17, Theorem 1] proved for $A, B \geq 0$ that

$$
\left\|A^{\frac{s+t}{2}} B^{t} A^{\frac{s+t}{2}}\right\| \leq\left\|A^{\frac{s}{2}}\left(A^{\frac{r}{2}} B^{r} A^{\frac{r}{2}}\right)^{\frac{t}{r}} A^{\frac{s}{2}}\right\|, \quad 0 \leq t \leq r, \quad s \geq 0,
$$

which reduces to Araki-Cordes inequality [5] if $s=0$ and to Bebiano-Lemos-Providência inequality [4, Theorem 2.1] if $s=1$. When $0 \leq s \leq r \leq t$ and $r>0$, they also proved that (11) holds with the reverse inequality sign [17, Theorem 2].

In this last section, we observe that it is easy to extend the determinantal inequality

$$
\operatorname{det}\left(A^{2}+|B A|\right) \leq \operatorname{det}\left(A^{2}+A B\right)
$$

formulated by K. M. R. Audenaert [1] for $A, B \geq 0$. This is a reformulation of the inequality

$$
\operatorname{det}\left(A+U^{*} B\right) \leq \operatorname{det}(A+B)
$$

where $U$ is a unitary matrix that appears in the polar decomposition of $B A$ for $A, B \geq 0$. According to Audenaert [1], this determinantal inequality has arisen in the study of interpolation methods for image processing in diffusion tensor imaging, when comparing geodesics induced by different metrics.

M. Lin [13] obtained a slightly more general inequality for $A, B \geq 0$, namely,

$$
\operatorname{det}\left(A^{2}+|B A|^{t}\right) \leq \operatorname{det}\left(A^{2}+A^{t} B^{t}\right), \quad 0 \leq t \leq 2 .
$$

In order to further extend it, we recall the norm inequality

$$
\left\|A^{\frac{c}{2}}\left(A^{-\frac{1}{2}} B A^{-\frac{1}{2}}\right)^{\alpha} A^{\frac{c}{2}}\right\| \leq\left\|A^{\frac{c-\alpha}{2}} B^{\alpha} A^{\frac{c-\alpha}{2}}\right\|, \quad 0 \leq \alpha \leq 1, \quad c \geq \alpha,
$$

for $A>0, B \geq 0$, obtained in [7, Corollary 3.1 (iii)], using Furuta inequality [6]. Replacing $A, B$ by $A^{-r}, B^{r}$, respectively, considering $\alpha=\frac{t}{r}$ and $c=-\frac{s}{r}$ in (14) yields the reverse of (11) for $0 \leq t \leq r$ and $-s \geq t$. As a consequence, we have the following extension of the determinantal inequality (13).

Proposition 4.1. Let $A>0$ and $B \geq 0$. Then

$$
\operatorname{det}\left(A^{-s}+\left(A^{\frac{r}{2}} B^{r} A^{\frac{r}{2}}\right)^{\frac{t}{r}}\right) \leq \operatorname{det}\left(A^{-s}+A^{t} B^{t}\right)
$$

holds if either (i) $0 \leq t \leq r$ and $-s \geq t$ or (ii) $0 \leq s \leq r \leq t$ with $r>0$; the reverse inequality holds if (iii) $0 \leq t \leq r$ and $s \geq 0$; occuring equality if $s \in \mathbb{R}$ and either $t=0$ or $t=r$. 
Proof. The norm inequality (11) implies the determinantal inequality

$$
\operatorname{det}\left(I+A^{s+t} B^{t}\right) \leq \operatorname{det}\left(I+A^{s}\left(A^{\frac{r}{2}} B^{r} A^{\frac{r}{2}}\right)^{\frac{t}{r}}\right), \quad 0 \leq t \leq r, \quad s \geq 0,
$$

occuring equality if $s \in \mathbb{R}$ and either $t=0$ or $t=r$. On the other hand, the norm inequality (11) holds with the reverse sign, which implies the reverse of the previous determinantal inequality, in the cases (i) $0 \leq s \leq r \leq t$ with $r>0$; (ii) $0 \leq t \leq r$ and $-s \geq t$. As observed previously, we find the case (ii) as a consequence of (14). Hence, the result follows, multiplying both hand sides of the previous inequalities by $\operatorname{det}\left(A^{-s}\right)>0$.

The particular case $r=2$ in Proposition 4.1 includes, for $A>0$ and $B \geq 0$, the inequality

$$
\operatorname{det}\left(A^{-s}+|B A|^{t}\right) \leq \operatorname{det}\left(A^{-s}+A^{t} B^{t}\right)
$$

if either $0 \leq t \leq 2$ and $-s \geq t$ or $0 \leq s \leq 2 \leq t$, as well as the reverse inequality if $0 \leq t \leq 2$ and $s \geq 0$. The case $s=-2$ yields (13) due to M. Lin.

Remark. The norm inequality (14) due to Furuta can be restated as follows. If $A>0, B \geq 0$, $0<\alpha<1$ and $c \geq \alpha$, then

$$
A \natural_{c, \alpha} B \prec_{\log } A^{c-\alpha} B^{\alpha},
$$

which holds trivially if $c \in \mathbb{R}$ and either $\alpha=0$ or $\alpha=1$. This log-majorization was obtained by J. S. Matharu and J. S. Aujla [16, Theorem 2.10] if $c=1$ and by D. T. Hoa [10, Proposition 2.1] if $c=2$. If $\alpha=\frac{1}{2}$ and $c=2$, the particular case

$$
A^{\frac{1}{2}}(A \sharp B) A^{\frac{1}{2}} \prec_{\log } A^{\frac{3}{4}} B^{\frac{1}{2}} A^{\frac{3}{4}}
$$

was considered by Bhatia, Lim and Yamazaki [3, Theorem 2]. Further, we observe that the case $r=1, s=-c, t=\alpha$, replacing $A$ by $A^{-1}$, in the norm inequality (11) and in its reverse by Matsumoto, Nakamoto and Fujii, yields (15) with the reverse log-majorization sign if $0 \leq \alpha \leq 1$ and $c \leq 0$; and again (15), whenever $\alpha \geq 1$ and $-1 \leq c \leq 0$.

If $A, B$ are density matrices, that is, positive semidefinite matrices of trace one, then

$$
S(A, B)=\operatorname{Tr}(A(\log A-\log B))
$$

is the Umegaki relative entropy [19] of $A, B$. Fujii and Kamei introduced the variant

$$
\hat{S}(A \mid B)=A^{\frac{1}{2}} \log \left(A^{-\frac{1}{2}} B A^{-\frac{1}{2}}\right) A^{\frac{1}{2}},
$$

that is, the connection associated to the logarithmic function. A logarithmic trace inequality is now presented, inspired by the corresponding case $s=t=1$ by F. Hiai and D. Petz [8].

Proposition 4.2. Let $A, B \geq 0$. If $t, s \geq 0$, then

$$
\operatorname{Tr}\left(A^{s}\left(\log A^{t}+\log B^{t}\right)\right) \leq \operatorname{Tr}\left(A^{s} \log \left(A^{\frac{r}{2}} B^{r} A^{\frac{r}{2}}\right)^{\frac{t}{r}}\right), \quad r>0,
$$

and the LHS converges to the RHS as $r \downarrow 0$.

Proof. The norm inequality (11) implies the trace inequality

$$
\operatorname{Tr}\left(A^{s} A^{t} B^{t}\right) \leq \operatorname{Tr}\left(A^{s}\left(A^{\frac{r}{2}} B^{r} A^{\frac{r}{2}}\right)^{\frac{t}{r}}\right), \quad 0 \leq t \leq r, \quad s \geq 0,
$$

occuring trace equality when $t=0$. Taking the derivatives of the RHS and LHS of the previous inequality at $t=0$, observing that

$$
\begin{gathered}
\left.\frac{d}{d t}\left(A^{t} B^{t}\right)\right|_{t=0}=\log A+\log B, \\
\left.\frac{d}{d t}\left(\left(A^{\frac{r}{2}} B^{r} A^{\frac{r}{2}}\right)^{\frac{t}{r}}\right)\right|_{t=0}=\log \left(A^{\frac{r}{2}} B^{r} A^{\frac{r}{2}}\right)^{\frac{1}{r}}, \quad r>0,
\end{gathered}
$$


yields a trace inequality. Multiplying both hand sides of the obtained trace inequality by $t \geq 0$, provides (16). By the parametric Lie-Trotter formula (see, for instance, [2, Exercise IX.1.5]), we may see that (18) converges to (17) as $r \downarrow 0$, that is, the convergence of the LHS of (16) to its RHS holds.

Using relative entropy terminology, the case $t=s$ of Proposition 4.2 , replacing $B$ by $B^{-1}$, may be written in the condensed form

$$
S\left(A^{s}, B^{s}\right) \leq-\frac{s}{r} \operatorname{Tr}\left(\hat{S}\left(A^{r} \mid B^{r}\right) A^{s-r}\right), \quad s \geq 0, \quad r>0
$$

this providing an upper bound for the relative entropy $S(A, B)$, when $s=1$.

If $r=s=2$ in Proposition 4.2, then we have the trace inequality

$$
\operatorname{Tr}\left(A^{2}(\log A+\log B)\right) \leq \operatorname{Tr}\left(A^{2} \log |B A|\right)
$$

in a parallel line to Audenaert's determinantal inequality (12), which motivated the considerations of this last section.

Acknowledgements The authors are grateful to the referee for careful reading of the manuscript and for useful comments. The work of the first author was supported by Portuguese funds through the Center for Research and Development in Mathematics and Applications (CIDMA) and the Portuguese Foundation for Science and Technology (FCT - Fundação para a Ciência e a Tecnologia), within the project UID/MAT/0416/2013. The work of the second author was financed by Portuguese Funds through FCT- Fundação para a Ciência e Tecnologia, within the Project UID/MAT/00013/2013.

\section{REFERENCES}

[1] K. M. R. Audenaert, A determinantal inequality for the geometric mean with an application in diffusion tensor imaging, http://arxiv.org/abs/1502.06902, 2015.

[2] R. Bhatia, Matrix Analysis, Springer, New York, 1997.

[3] R. Bhatia, Y. Lim and T. Yamazaki, Some norm inequalities for matrix means, Linear Algebra Appl. 501 (2016), 112-122.

[4] N. Bebiano, R. Lemos and J. da Providência, Inequalities for quantum relative entropy, Linear Algebra Appl. 401 (2005), 159-172.

[5] J. I. Fujii and T. Furuta, Löwner-Heinz, Cordes and Heinz-Kato inequalities, Math. Japon. 38 (1993), 73-78.

[6] T. Furuta, $A \geq B \geq 0$ assures $\left(B^{r} A^{p} B^{r}\right)^{1 / q} \geq B^{\{p+2 r) / q}$ for $r \geq 0, p \geq 0, q \geq 1$ with $(1+2 r) q \geq p+2 r$, Proc. Amer. Math. Soc. 101 (1987), 85-88.

[7] T. Furuta, Extensions of inequalities for unitarily invariant norms via log majorization, Linear Algebra Appl. 436 (2012), 3463-3468.

[8] F. Hiai and D. Petz, The Golden-Thompson trace inequality is complemented, Linear Algebra Appl. 181 (1993), 153-185.

[9] F. Hiai and M. Lin, On an eigenvalue inequality involving the Hadamard product, Linear Algebra Appl. 515 (2017), 313-320.

[10] D. T. Hoa, Some inequalities for the matrix Heron mean, Linear Algebra Appl. 528 (2017), 321-330.

[11] R. A. Horn and C. R. Johnson, Matrix analysis, Cambridge University Press, Cambridge, 1985.

[12] F. Kubo and T. Ando, Means of positive linear operators, Math. Ann. 246 (1980), 205-224.

[13] M. Lin, On a determinantal inequality arising from diffusion tensor imaging, Commun. Contemp. Math. 19, $\mathrm{n}^{\circ} 5$ (2017), 6 pp.

[14] K. Löwner, Über monotone Matrixfunktionen, Math. Z. 38 (1934), 177-216.

[15] A. W. Marshall and I. Olkin, Inequalities: Theory of Majorization and its Applications, Academic, New York, 1979.

[16] J. S. Matharu and J. S Aujla, Some inequalities for unitarily invariant norms, Linear Algebra Appl. 436 (2012), 1623-1631.

[17] A. Matsumoto, R. Nakamoto and M. Fujii, Reverse of Bebiano-Lemos-Providencia inequality and Complementary Furuta inequality (Inequalities on Linear Operators and its Applications), Departmental Bulletin Paper, Kyoto University (2008), 91-98. 
[18] W. Pusz and S. L. Woronowicz, Functional calculus for sesquilinear forms and the purification map, Reo. Math. Phys. 8 (1975), 159-170.

[19] H. Umegaki, Condition expectation in an operator algebra IV, Kodai Math. Sem. Rep. 14 (1962), 59-85.

[20] L. Zou, An arithmetic geometric mean inequality for singular values and its applications, Linear Algebra Appl. 521 (2017), 25-32.

(Rute Lemos) Cidma, Mathematics Department, University of Aveiro, 3810-193 Aveiro, PorTUGAL

E-mail address, Rute Lemos: rute@ua.pt

(Graça Soares) Mathematics Center CMAT, Pole CMAT-Utad, CEMAT-IST-UL, Universidade de Trás-os-Montes e Alto Douro, Utad, Escola das Ciências e Tecnologia, Quinta dos Prados, 5000-801 Vila Real, Portugal, www.utad.Pt

E-mail address, Graça Soares: gsoares@utad.pt 